\title{
Signal and backgrounds for the single production of scalar and vector leptoquarks at the CERN LHC
}

\author{
J. E. Cieza Montalvo, ${ }^{1}$ O. J. P. Éboli, ${ }^{2,3}$ M. B. Magro, ${ }^{3}$ and P. G. Mercadante ${ }^{4}$ \\ ${ }^{1}$ Instituto de Física, Universidade do Estado do Rio de Janeiro, CEP 20559-900 Rio de Janeiro, Brazil \\ ${ }^{2}$ Instituto de Física Teórica, Universidade Estadual Paulista, Rua Pamplona 145, São Paulo, SP 01405, Brazil \\ ${ }^{3}$ Department of Physics, University of Wisconsin, Madison, Wisconsin 53706 \\ ${ }^{4}$ Department of Physics \& Astronomy, University of Hawaii, Honolulu, Hawaii 96822
}

(Received 28 May 1998; published 15 September 1998)

\begin{abstract}
We perform a detailed analysis of the potentiality of the CERN Large Hadron Collider to study the single production of leptoquarks via $p p \rightarrow e^{ \pm} q \rightarrow$ leptoquark $\rightarrow e^{ \pm} q$, with $e^{ \pm}$generated by the splitting of photons radiated by the protons. Working with the most general $\mathrm{SU}(2)_{L} \otimes \mathrm{U}(1)_{Y}$ invariant effective Lagrangian for scalar and vector leptoquarks, we analyze in detail the leptoquark signals and backgrounds that lead to a final state containing an $e^{ \pm}$and a hard jet with approximately balanced transverse momenta. Our results indicate that the LHC will be able to discover leptoquarks with masses up to $2-3 \mathrm{TeV}$, depending on their type, for Yukawa couplings of the order of the electromagnetic one.
\end{abstract}

[S0556-2821(98)08219-8]

PACS number(s): 12.60.-i, 13.85.Rm, 14.80.-j

\section{INTRODUCTION}

Leptoquarks, which are particles that carry simultaneously leptonic and baryonic numbers, provide a clear sign for many extensions of the standard model (SM) that treat quarks and leptons on the same footing. There are many models exhibiting these new particles, such as technicolor [1], composite models [2,3], grand unified theories [4], and superstring-inspired models [5]. From the experimental point of view, leptoquarks possess the striking signature of a peak in the invariant mass of a charged lepton with a jet, which makes their search much simpler without the need of elaborate analyses of several final state topologies.

Pair production of leptoquarks in a hadronic collider takes place via quark-quark and gluon-gluon fusions, being essentially model independent since the leptoquark-gluon interaction is fixed by the $\mathrm{SU}(3)_{C}$ gauge invariance, with the only free parameter being an "anomalous chromomagetic moment" for vector leptoquarks. On the other hand, single production is model dependent because it takes place via leptoquark interactions with quarks and leptons. Notwithstanding, these two signals are complementary because they allow us not only to reveal the existence of leptoquarks but also to determine their properties such as mass and Yukawa couplings to quarks and leptons.

The direct search for leptoquarks with masses above a few hundred GeV can be carried out only in the next generation of colliders. In fact, there have been many studies of the production of leptoquarks in the future $p p[6]$, ep $[7,8]$, $e^{+} e^{-}$[9], $e^{-} e^{-}$[10], e $\gamma$ [11], and $\gamma \gamma$ [12] colliders. In particular, the usual studies for leptoquarks in hadronic colliders concentrated on the processes [13]

$$
\begin{aligned}
& q+g \rightarrow \Phi_{\mathrm{lq}}+l, \\
& q+\bar{q} \rightarrow \Phi_{\mathrm{lq}}+\Phi_{\mathrm{lq}},
\end{aligned}
$$

$$
g+g \rightarrow \Phi_{\mathrm{lq}}+\bar{\Phi}_{\mathrm{lq}}
$$

where $l=e^{ \pm}\left(\mu^{ \pm}\right)$and we denoted scalar and vector leptoquarks by $\Phi_{\text {lq }}$. These processes give rise to $e^{+} e^{-}$pairs with large transverse momenta accompanied by one or more jets.

In this work, we study the capability of the CERN Large Hadron Collider (LHC) to unravel the existence of first generation leptoquarks through the final state topology jet plus $e^{ \pm}$. This process was first analyzed in Ref. [14], and it occurs via

$$
p p \rightarrow e^{ \pm} q \rightarrow \text { leptoquark } \rightarrow e^{ \pm} q,
$$

where the $e^{ \pm}$originates from the splitting of a photon radiated by a quark. This reaction leads an $e^{ \pm}$-jet pair with balanced transverse momenta, up to the detector resolution. This feature allows us to separate the production mechanisms (1)-(3) from the above reaction. Therefore, this process provides one more handle to study the leptoquark properties.

We performed a careful analysis of the signal and its respective backgrounds for leptoquarks that couple to pairs $e^{-} u, e^{+} u, e^{-} d$, or $e^{+} d$, assuming the most general effective Lagrangian that is invariant under $\mathrm{SU}(3)_{C} \otimes \mathrm{SU}(2)_{L}$ $\otimes \mathrm{U}(1)_{Y}[7]$. We studied not only a series of cuts to reduce the backgrounds, but also strategies to discriminate among the several leptoquark types. Our analysis improves the previous one [14] since we considered all possible backgrounds as well as the most general model for leptoquarks.

We show in this work that the single leptoquark search at the LHC can discover, at the $3 \sigma$ level, leptoquarks with masses up to 2-3 TeV, depending on their type, for an integrated luminosity of $100 \mathrm{fb}^{-1}$, a center-of-mass energy of $14 \mathrm{TeV}$, and Yukawa couplings of the order of the electromagnetic one. We also exhibit the region of the Yukawa coupling and leptoquark mass plane that can be ruled out at the LHC. 
The outline of this paper is as follows. In Sec. II we introduce the $\mathrm{SU}(2)_{L} \otimes \mathrm{U}(1)_{Y}$ invariant effective Lagrangians that we analyzed and present the available bounds on leptoquarks. Section III contains a detailed description of the leptoquark signal and its backgrounds, as well as the cuts used to enhance the signal. We present our results in Sec. IV and draw our conclusions in Sec. V.

\section{MODELS FOR LEPTOQUARK INTERACTIONS}

A natural hypothesis for theories beyond the SM is that they exhibit the gauge symmetry $\mathrm{SU}(2)_{L} \otimes \mathrm{U}(1)_{Y}$ above the electroweak symmetry breaking scale $v$. Therefore, we imposed this symmetry on the leptoquark interactions. Moreover, in order to avoid strong bounds coming from the proton lifetime experiments, we required baryon $(B)$ and lepton $(L)$ number conservation, which forbids the leptoquarks to couple to pairs of quarks or leptons. The most general effective Lagrangian for scalar and vector leptoquarks satisfying the above requirements and electric charge and color conservation is given by [7]

$$
\begin{aligned}
\mathcal{L}_{\text {eff }}= & \mathcal{L}_{F=2}+\mathcal{L}_{F=0}+\text { H.c. } \\
\mathcal{L}_{F=2}= & g_{1 L} \bar{q}_{L}^{c} i \tau_{2} l_{L} S_{1 L}+g_{1 R} \bar{u}_{R}^{c} e_{R} S_{1 R} \\
& +\tilde{g}_{1 R} \bar{d}_{R}^{c} e_{R} \widetilde{S}_{1}+g_{3 L} \bar{q}_{L}^{c} i \tau_{2} \vec{\tau} l_{L} \cdot \vec{S}_{3} \\
& +g_{2 L}\left(V_{2 \mu}^{L}\right)^{T} \bar{d}_{R}^{c} \gamma^{\mu} i \tau_{2} l_{L} \\
& +g_{2 R} \bar{q}_{L}^{c} \gamma^{\mu} i \tau_{2} e_{R} V_{2 \mu}^{R} \\
& +\tilde{g}_{2 L}\left(\widetilde{V}_{2 \mu}^{L}\right)^{T} \bar{u}_{R}^{c} \gamma^{\mu} i \tau_{2} l_{L}, \\
\mathcal{L}_{F=0}= & h_{2 L} R_{2 L}^{T} \bar{u}_{R} i \tau_{2} l_{L}+h_{2 R} \bar{q}_{L} e_{R} R_{2 R} \\
& +\widetilde{h}_{2 L} \widetilde{R}_{2}^{T} \bar{d}_{R} i \tau_{2} l_{L}+h_{1 L} \bar{q}_{L} \gamma^{\mu} l_{L} U_{1 \mu}^{L} \\
& +h_{1 R} \bar{d}_{R} \gamma^{\mu} e_{R} U_{1 \mu}^{R}+\tilde{h}_{1 R} \bar{u}_{R} \gamma^{\mu} e_{R} \widetilde{U}_{1 \mu}^{R} \\
& +h_{3 L} \bar{q}_{L} \vec{\tau}^{\mu} l_{L} \vec{U}_{3 \mu}^{L},
\end{aligned}
$$

where $F=3 B+L, q_{L}\left(l_{L}\right)$ stands for the left-handed quark (lepton) doublet, and $u_{R}, d_{R}$, and $e_{R}$ are the singlet components of the fermions. We denoted the charge-conjugated fermion fields by $\psi^{c}=C \bar{\psi}^{T}$ and we omitted in Eqs. (6) and (7) the flavor indices of the leptoquark couplings to fermions. The leptoquarks $S_{1 R(L)}, \widetilde{S}_{1}, U_{1 \mu}^{L(R)}$, and $\widetilde{U}_{1 \mu}^{R}$ are singlets under $\mathrm{SU}(2)_{L}$, while $R_{2 R(L)}, \widetilde{R}_{2}, V_{2 \mu}^{R(L)}$, and $\widetilde{V}_{2 \mu}^{L}$ are doublets, and $S_{3}$ and $\vec{U}_{3 \mu}^{L}$ are triplets. The quantum numbers for all leptoquarks can be found, for instance, in the last reference of [9]. In this work, we denoted the Yukawa couplings $h$ and $g$ by $\kappa$.

\begin{tabular}{|c|c|c|c|}
\hline$L Q$ & $Q_{e m}$ & Decay channels & Coupling $\left(\kappa_{L / R}\right)$ \\
\hline \multirow[t]{2}{*}{$S_{1}$} & $-1 / 3$ & $d \nu_{e}$ & $-g_{1 L}$ \\
\hline & $-1 / 3$ & $u e^{-}$ & $g_{1 L} ; g_{1 R}$ \\
\hline$\widetilde{S}_{1}$ & $-4 / 3$ & $d e^{-}$ & $\tilde{g}_{1 R}$ \\
\hline$S_{3}^{+}$ & $2 / 3$ & $u \nu_{e}$ & $\sqrt{2} g_{3 L}$ \\
\hline$S_{3}^{-}$ & $-4 / 3$ & $d e^{-}$ & $\sqrt{2} g_{3 L}$ \\
\hline \multirow[t]{3}{*}{$S_{3}^{0}$} & $-1 / 3$ & $d \nu_{e}$ & $-g_{3 L}$ \\
\hline & $-1 / 3$ & $u e^{-}$ & $-g_{3 L}$ \\
\hline & $-4 / 3$ & $d e^{-}$ & $g_{2 L} ;-g_{2 R}$ \\
\hline \multirow[t]{2}{*}{$V_{2 \mu}$} & $-1 / 3$ & $u e^{-}$ & $g_{2 R}$ \\
\hline & $-1 / 3$ & $d \nu_{e}$ & $-g_{2 L}$ \\
\hline \multirow[t]{3}{*}{$\widetilde{V}_{2 \mu}$} & $-1 / 3$ & $u e^{-}$ & $\tilde{g}_{2 L}$ \\
\hline & $2 / 3$ & $u \nu_{e}$ & $-\tilde{g}_{2 L}$ \\
\hline & $5 / 3$ & $u e^{+}$ & $h_{2 L} ; h_{2 R}$ \\
\hline \multirow[t]{2}{*}{$R_{2}$} & $2 / 3$ & $u \bar{\nu}_{e}$ & $h_{2 L}$ \\
\hline & $2 / 3$ & $d e^{+}$ & $-h_{2 R}$ \\
\hline \multirow[t]{2}{*}{$\widetilde{R}_{2}$} & $-1 / 3$ & $d \bar{\nu}_{e}$ & $\tilde{h}_{2 L}$ \\
\hline & $2 / 3$ & $d e^{+}$ & $\tilde{h}_{2 L}$ \\
\hline \multirow[t]{2}{*}{$U_{1 \mu}$} & $2 / 3$ & $u \bar{\nu}_{e}$ & $h_{1 L}$ \\
\hline & $2 / 3$ & $d e^{+}$ & $h_{1 L} ; h_{1 R}$ \\
\hline$\widetilde{U}_{1 \mu}$ & $5 / 3$ & $u e^{+}$ & $\widetilde{h}_{1 R}$ \\
\hline$U_{3}^{+\mu}$ & $-1 / 3$ & $d \bar{\nu}_{e}$ & $\sqrt{2} h_{3 L}$ \\
\hline$U_{3}^{-\mu}$ & $5 / 3$ & $u e^{+}$ & $\sqrt{2} h_{2 L}$ \\
\hline \multirow[t]{2}{*}{$U_{3}^{0 \mu}$} & $2 / 3$ & $u \bar{\nu}_{e}$ & $h_{3 L}$ \\
\hline & $2 / 3$ & $d e^{+}$ & $-h_{3 L}$ \\
\hline
\end{tabular}

We can see from the above interactions that the main decay modes of leptoquarks are into pairs $e^{ \pm} q$ and/or $\nu_{e} q^{\prime}$; thus, their signal is either a $e^{ \pm}$plus a jet or a jet plus missing energy. However, this is true provided the leptoquark masses are such that they cannot decay into another leptoquark be-
TABLE I. Scalar and vector leptoquarks that can be observed through their decays into a $e^{ \pm}$and a jet and the correspondent decay channels. For simplicity we introduced the left- and righthanded leptoquarks in the same entry.

longing to the same multiplet and a vector boson. Here we assumed that the leptoquarks belonging to the same multiplet are degenerate in mass. Furthermore, we implicitly assumed that the leptoquarks couple only to the known particles; i.e., we do not consider the scenario where the leptoquarks also couple to other new particles like charginos or neutralinos in $R$-parity violating SUSY models.

In this work we considered only the $e^{ \pm} q$ decay mode and took into account the corresponding branching ratio. We exhibit in Table I the leptoquarks that can be analyzed using the final state $e^{ \pm}$plus a jet, as well as their decay products. As we can see from Eqs. (6) and (7), only the leptoquarks $R_{2 L}^{2}, \widetilde{R}_{2}^{1}, S_{3}^{+}, V_{2}^{2 \mu}$, and $U_{3}^{+\mu}$ decay exclusively into a jet and a neutrino.

There have been many searches for leptoquarks which, so far, have led to negative results. Analyzing the decay of the $Z$ into a pair of on-shell leptoquarks, the CERN $e^{+} e^{-}$collider LEP experiments established a lower bound $M_{\mathrm{lq}} \geqslant 44 \mathrm{GeV}$ for scalar leptoquarks $[15,16]$. Recently the LEP Collaborations [17] used their $\sqrt{s}=161$ and $172 \mathrm{GeV}$ data to obtain the constraint $M_{\mathrm{lq}} \gtrsim 131 \mathrm{GeV}$ for leptoquarks coupling to the first family quarks and electrons. The search for scalar (vector) leptoquarks decaying exclusively into electron-jet pairs at the Tevatron constrained their masses to be $M_{\mathrm{lq}} \geqslant 225$ 
(240) $\mathrm{GeV}$ [18]. Furthermore, the experiments at the DESY $e p$ collider HERA [19] placed limits on their masses and couplings, establishing that $M_{\mathrm{lq}} \geqslant 216-275 \mathrm{GeV}$ depending on the leptoquark type and couplings.

Low-energy experiments also lead to strong indirect bounds on the couplings and masses of leptoquarks, which can be used to define the goals of new machines to search for these particles. The main sources of indirect constraints are the following.

(i) Leptoquarks give rise to flavor changing neutral current (FCNC) processes if they couple to more than one family of quarks or leptons $[20,21]$. In order to avoid strong bounds from FCNC, we assumed that the leptoquarks couple to a single generation of quarks and a single one of leptons. However, because of mixing effects on the quark sector, there is still some amount of FCNC left [22] and, therefore, leptoquarks that couple to the first two generations of quarks must comply with some low-energy bounds [22].

(ii) The analyses of the decays of pseudoscalar mesons, such as the pions, put stringent bounds on leptoquarks unless their coupling is chiral-that is, it is either left handed or right handed [20].

(iii) Leptoquarks that couple to the first family of quarks and leptons are strongly constrained by atomic parity violation [23]. In this case, there is no choice of couplings that avoids the strong limits.

(iv) The analyses of the effects of leptoquarks on the $Z$ physics through radiative corrections lead to limits on the masses and couplings of leptoquarks that couple to top quarks $[24,25]$.

As a rule of a thumb, the low-energy data constrain the masses of leptoquarks to be larger than $0.5-1 \mathrm{TeV}$ when their Yukawa coupling is equal to the electromagnetic coupling $e[22,25,26]$. Therefore, our results indicate that the LHC cannot only confirm these indirect limits but also expand them considerably.

\section{SIGNALS AND BACKGROUNDS FOR THE PRODUCTION OF LEPTOQUARKS}

In this work we focus our attention on the $s$-channel leptoquark production via

$$
p p \rightarrow q q \rightarrow \gamma q \rightarrow e^{ \pm} q \rightarrow \Phi_{\mathrm{lq}} \rightarrow e^{ \pm} q,
$$

which leads to $e^{ \pm}$-jet pairs with balanced transverse momenta, up to the detector resolution. In our evaluation of the subprocess cross section $e q \rightarrow e q$, we included the irreducible SM background due to the $\gamma$ and $Z$ exchange, treating properly its interference with the leptoquark diagrams; see Fig. 1. The expressions for the subprocess cross sections $\left(\hat{\sigma}_{e q \rightarrow e q}\right)$ are presented in the Appendix for all leptoquark models. The leptoquark production cross section is then obtained by folding $\hat{\sigma}_{e q \rightarrow e q}$ with the quark $\left(f_{q / p}\right)$ and $e^{ \pm}\left(f_{e / p}\right)$ distributions in the proton:

$$
\sigma(p p \rightarrow e q X)=\int d x_{e} d x_{q} f_{e / p}\left(x_{e}\right) f_{q / p}\left(x_{q}\right) \hat{\sigma}_{e q \rightarrow e q}(\hat{s}),
$$
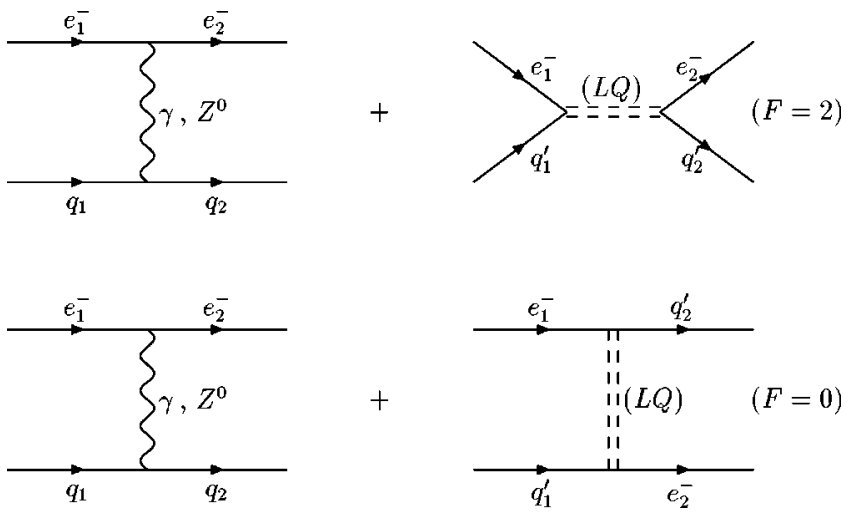

FIG. 1. Feynman diagrams that contribute to the process $e^{-} q$ $\rightarrow e^{-} q$, with $q_{i}=u, d, s, c$ and $q_{i}^{\prime}=u, d$. We denoted the scalar and vector leptoquarks by $L Q$.

where the subprocess center-of-mass energy $(\sqrt{\hat{s}})$ is related to the $p p$ one $(\sqrt{s})$ by $\hat{s}=x_{e} x_{q} s$.

The distribution of $e^{ \pm}$in the proton is given by

$$
f_{e / p}\left(x_{e}\right)=\int_{x_{e}}^{1} \frac{d z}{z} f_{e / \gamma}(z) f_{\gamma / p}\left(\frac{x_{e}}{z}\right),
$$

with $f_{\gamma / p}$ being the distribution of photons in the proton and the splitting rate of $\gamma$ into $e^{+} e^{-}$pairs given by [27]

$$
f_{e / \gamma}(z)=\frac{\alpha}{2 \pi}\left[z^{2}+(1-z)^{2}\right] \log \left(\frac{Q^{2}}{m_{e}^{2}}\right) .
$$

We chose the scale $Q^{2}=M_{l q}^{2}$ and denoted by $m_{e}\left(M_{\mathrm{lq}}\right)$ the electron (leptoquark) mass. There are two possibilities for radiation photons of the proton: either the photons are radiated by the proton as a whole and it does not break off, or quarks radiate the photons and the proton fragments. Since this last mechanism leads to a larger photon flux, we considered only it in our analyses. In this case, the photon distribution in the proton is [14]

$f_{\gamma / p}\left(x_{\gamma}\right)=\frac{\alpha}{2 \pi} \log \left(\frac{Q^{2}}{m_{q}^{2}}\right) \frac{1}{x_{\gamma}} \int_{x_{\gamma}}^{1} \frac{d z}{z}\left[1+\left(1-x_{\gamma} / z\right)^{2}\right] F_{2}\left(z, Q^{2}\right)$,

with $F_{2}\left(z, Q^{2}\right)$ being the structure function of a quark inside the proton summed over all the quark flavors including the electric charge factors.

There are many SM processes that lead to the production of $e^{ \pm}$and jets. Since some of them give rise to more than one jet or $e^{ \pm}$, we can enhance the signal, demanding the presence of a single $e^{ \pm}$and a single jet in the central region of the detector. This requirement eliminates dangerous backgrounds like the pair production of electroweak gauge bosons or top quarks [13]. Nevertheless, there are further backgrounds for the leptoquark search like the scattering of $e^{ \pm}$and (anti)quarks with flavors different from the leptoquark ones; see Fig. 1. 

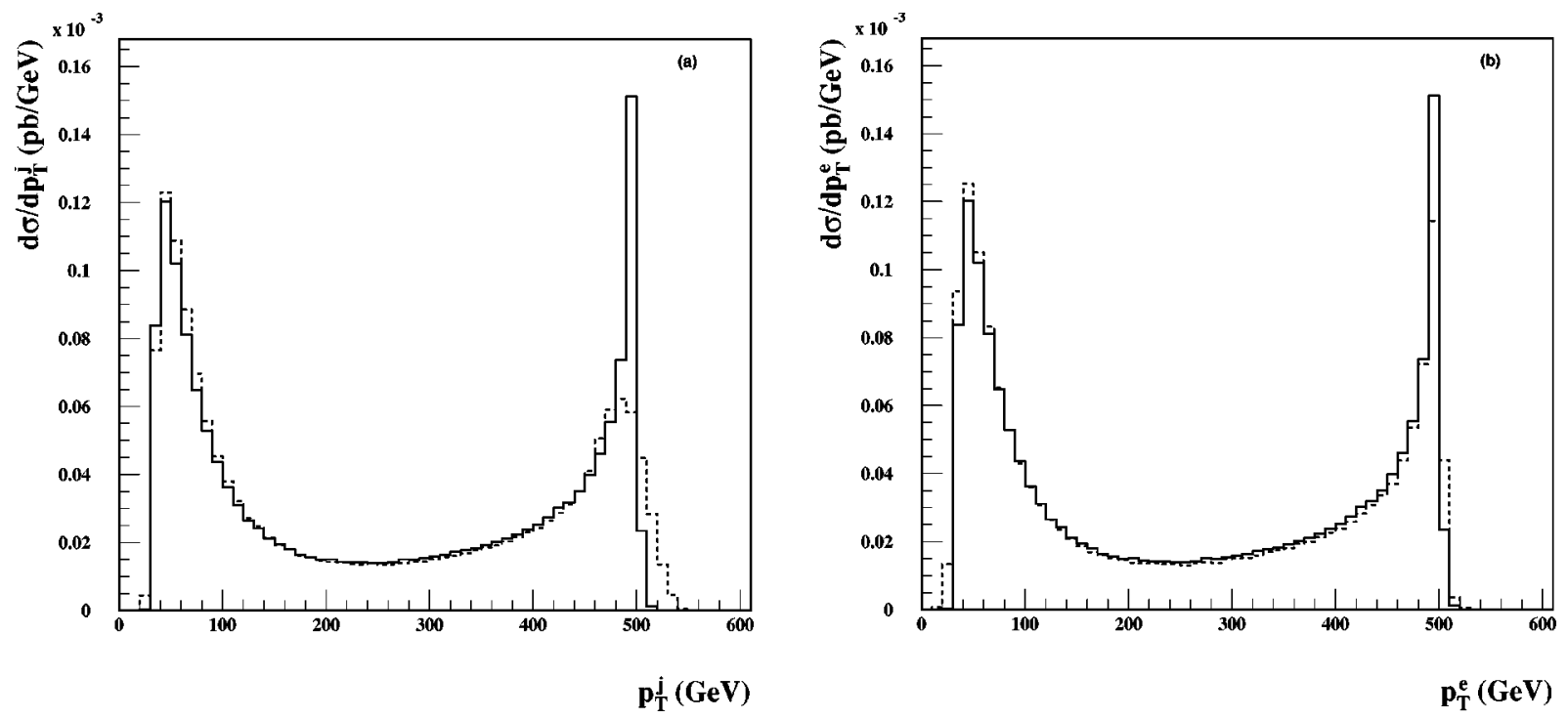

FIG. 2. $p_{T}$ distributions of the jet (a) and $e^{ \pm}$(b) originating from leptoquarks before (solid line) and after (dashed line) applying the calorimeter resolution for $M_{\mathrm{lq}}=1 \mathrm{TeV}$ and $\kappa=0.3$. We imposed the pseudorapidity cuts $\left|y_{e^{ \pm}, j}\right|<3.5$ and required the $e$-jet invariant mass to be in the range $\left|M_{\mathrm{lq}} \pm 40\right|(\mathrm{GeV})$.

In actual experiments, the observed signal events will not possess a balance between the $e^{ \pm}$and jet transverse momenta due to the experimental detector resolution. Consequently, we must analyze backgrounds like the $W$-jet production, where the $W$ decays into a pair electron-neutrino. Moreover, we should also consider the SM production of $Z$-jet pairs with the $Z$ decaying into a $e^{+} e^{-}$pair and one of the $e^{ \pm}$escaping undetected.

We mimicked the experimental resolution of the electromagnetic and hadronic calorimeters by smearing the final state quark and lepton energies according to

$$
\begin{aligned}
& \left.\frac{\delta E}{E}\right|_{\text {em }}=\frac{0.02}{\sqrt{E}} \oplus 0.005 \text { electromagnetic, } \\
& \left.\frac{\delta E}{E}\right|_{\text {had }}=\frac{0.6}{\sqrt{E}} \oplus 0.03 \text { hadronic. }
\end{aligned}
$$

Angles were smeared in a cone with

$$
\begin{aligned}
& \left.\delta \theta\right|_{\text {em }}=10 \mathrm{mrad} \text { electromagnetic, } \\
& \left.\delta \theta\right|_{\text {had }}=15 \mathrm{mrad} \text { hadronic. }
\end{aligned}
$$

We show in Figs. $2 \mathrm{a}$ and $2 \mathrm{~b}$ the typical behavior of the $p_{T}$ distributions of the jet and $e^{ \pm}$before (solid line) and after (dashed line) applying the calorimeter resolution for the process (8), including all $e q \rightarrow e q$ irreducible backgrounds; see Fig. 1. In these figures, we assumed a $S_{1 L}$ leptoquark with $M_{\mathrm{lq}}=1 \mathrm{TeV}$ and $\kappa=0.3$, and we also required that $\left|y_{e^{ \pm}, j}\right|$ $<3.5$ and the invariant mass of the $e-j$ pair $\left(M_{e j}\right)$ to be in the range $\left|M_{\mathrm{lq}} \pm 40\right| \mathrm{GeV}$. The peak around $p_{T}=M_{\mathrm{lq}} / 2$ is due to the leptoquark production while the low- $p_{T}$ peak is associated with the SM backgrounds. This feature of the $p_{T}$ spectrum provides an efficient way to separate the leptoquark signal from backgrounds. Moreover, the calorimeter resolution broadens the peak associated with the signal and increases slightly the low- $p_{T}$ peak associated with the $t$ channel backgrounds without changing significantly the total cross section.

We show in Fig. 3 the missing $p_{T}$ spectrum originating from the smearing of the momenta of the final state jet and $e^{ \pm}$in process (8), using the same parameters and cuts of Fig. 2. As expected, the missing $p_{T}$ distribution is peaked at small values, being negligible for missing $p_{T}$ 's larger than $70 \mathrm{GeV}$. Moreover, the missing $p_{T}$ in the signal events should be parallel to the total $p_{T}$ of the $e^{ \pm}$-jet system since the main

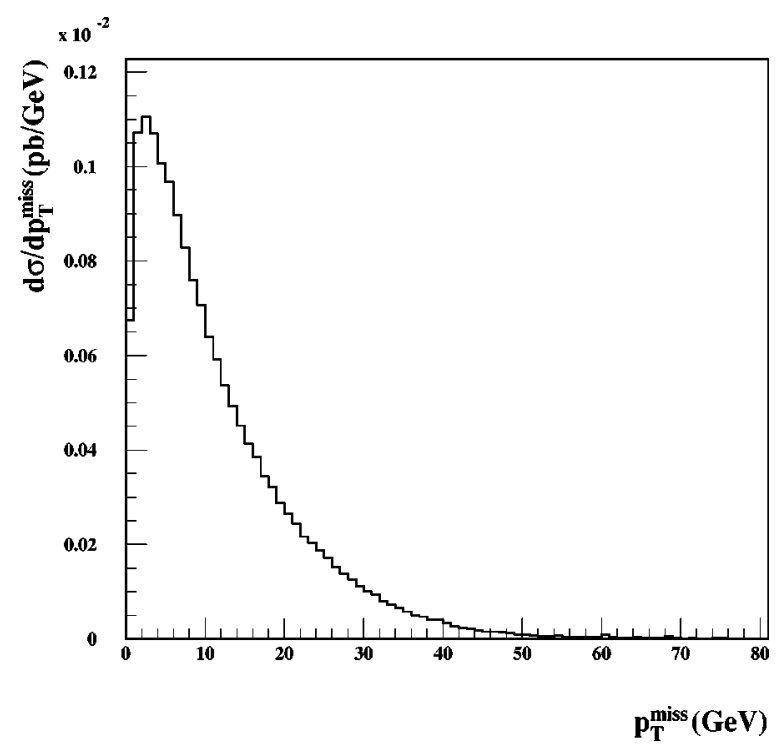

FIG. 3. Missing $p_{T}$ distribution in the process (8) due to the calorimeter resolution. We assumed the same parameters and cuts used in Fig. 2. 

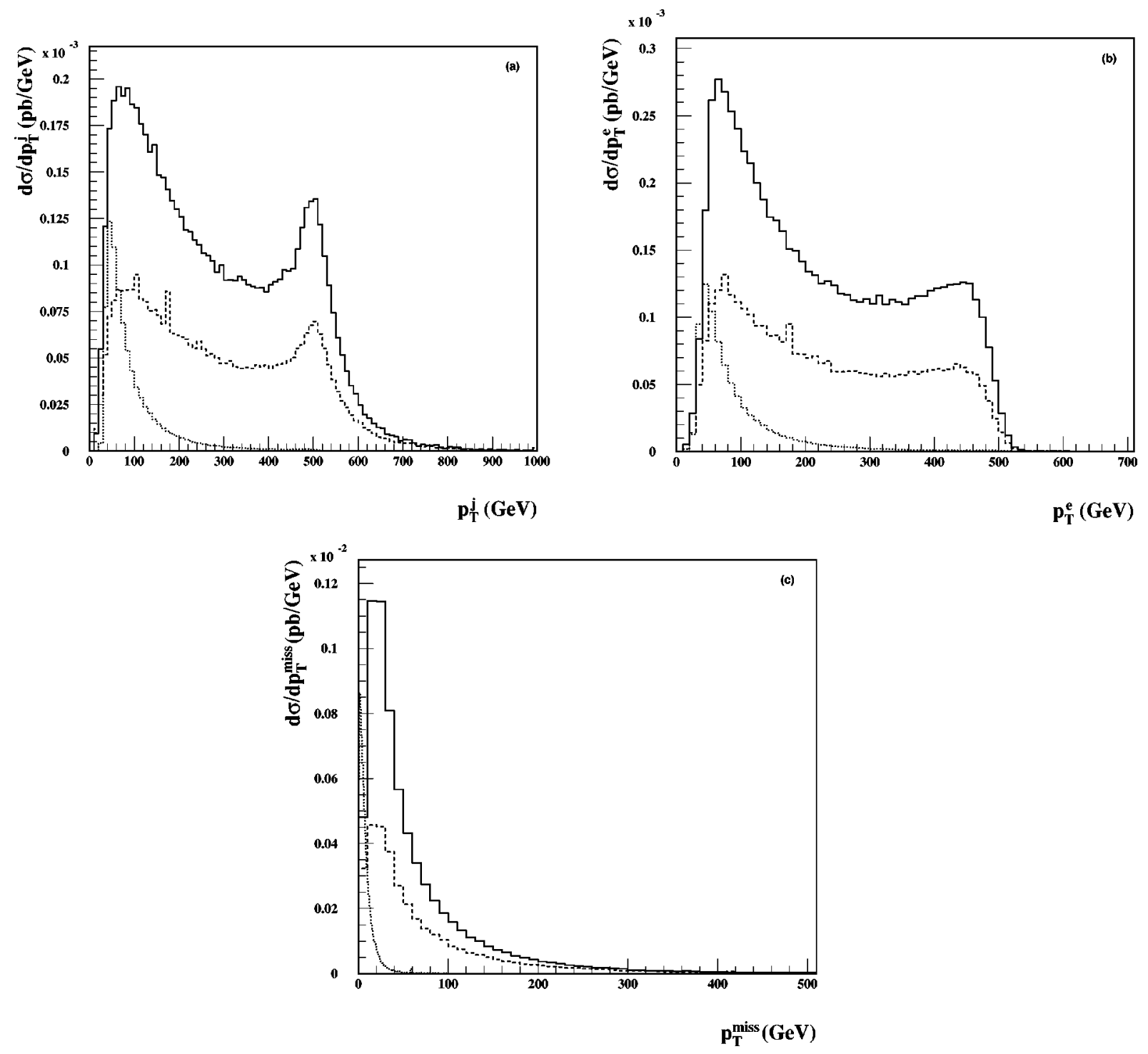

FIG. 4. Jet (a),$e^{ \pm}$(b), and missing (c) $p_{T}$ spectrum originating from the $W$-jet (solid line), $Z$-jet (dashed line), and $e q \rightarrow e q$ with $\kappa$ $=0$ (dotted line) backgrounds after applying the calorimeter resolution. We imposed the same cuts used in Fig. 2 and required $p_{T}^{j, e}$ $>10 \mathrm{GeV}$.

effect of the experimental resolution is to alter the magnitude of the measured transverse momenta.

We present in Fig. 4 several distributions for the backgrounds described above which should be contrasted with the signal ones. Figure $4 \mathrm{a}, 4 \mathrm{~b}$ contains the $p_{T}$ spectrum of the jet $\left(e^{ \pm}\right)$coming from the backgrounds after we applied the same cuts used in Fig. 2 and required $p_{T}^{j, e}>10 \mathrm{GeV}$ to avoid divergences due to gluons. As we can see, the backgrounds are peaked at low transverse momentum of the jet $\left(e^{ \pm}\right)$with the largest background being $W$-jet production. Despite the lack of a $s$-channel resonance, the $p_{T}$ spectra peak around $M_{\mathrm{lq}} / 2$ due to the $e^{ \pm}$-jet invariant mass cut. The missing $p_{T}$ distribution of the background, shown in Fig. 4c, reaches its maximum around $40 \mathrm{GeV}$ and extends up to $300 \mathrm{GeV}$ approximately, possessing a larger fraction of events at large missing $p_{T}$ than the signal.

Taking into account the above features of the signal and backgrounds we imposed the following set of cuts in order to enhance the signal and suppress the backgrounds.

(C1) The first requirement is that the jet and $e^{ \pm}$be in the pseudorapidity interval $|y|<3.5$.

(C2) We also demanded the events to have the $e$-jet invariant mass in the range $\left|M_{\mathrm{lq}} \pm \Delta M\right|$ with $\Delta M$ given in Table II.

(C3) We veto events exhibiting an extra $e^{ \pm}$(or parton) in

TABLE II. Values of the cuts $p_{T}^{\min }, \Delta M$, and $p_{T}^{\text {miss }}$ for several leptoquark masses.

\begin{tabular}{lcccccccc}
\hline \hline$M_{\mathrm{lq}}(\mathrm{GeV})$ & 500 & 750 & 1000 & 1250 & 1500 & 2000 & 2500 & 3000 \\
$p_{T}^{\min }(\mathrm{GeV})$ & 200 & 300 & 400 & 500 & 600 & 800 & 1000 & 1000 \\
$\Delta M(\mathrm{GeV})$ & 40 & 40 & 40 & 50 & 50 & 50 & 60 & 60 \\
$p_{T}^{\text {miss }}(\mathrm{GeV})$ & 30 & 30 & 30 & 60 & 60 & 60 & 80 & 80 \\
\hline \hline
\end{tabular}


TABLE III. Total cross section in fb for the signals and backgrounds for all leptoquark multiplets after and before applying the cuts (C3)-(C6). We assumed $M_{\mathrm{lq}}=1 \mathrm{TeV}$ and $\kappa=0.3$ and smeared all the final state momenta.

\begin{tabular}{lcccc}
\hline \hline & $e^{-}+$jet & \multicolumn{3}{c}{$e^{+}+$jet } \\
\hline & $(\mathrm{C} 1)+(\mathrm{C} 2)$ & $(\mathrm{C} 1)-(\mathrm{C} 6)$ & $(\mathrm{C} 1)+(\mathrm{C} 2)$ & $(\mathrm{C} 1)-(\mathrm{C} 6)$ \\
\hline$\widetilde{S}_{1}$ & 16.3 & 2.30 & 9.98 & 0.576 \\
$S_{1 L}$ & 16.9 & 2.45 & 9.02 & 0.299 \\
$S_{1 R}$ & 26.0 & 4.95 & 10.0 & 0.579 \\
$S_{3}$ & 32.9 & 6.86 & 12.9 & 1.37 \\
$V_{2 L}^{\mu}$ & 24.6 & 3.85 & 12.0 & 0.953 \\
$V_{2 R}^{\mu}$ & 60.6 & 12.1 & 16.0 & 1.88 \\
$\widetilde{V}_{2}^{\mu}$ & 44.1 & 8.32 & 12.1 & 0.957 \\
$\widetilde{R}_{2}$ & 10.1 & 0.595 & 16.2 & 2.28 \\
$R_{2 L}$ & 10.1 & 0.594 & 25.8 & 4.93 \\
$R_{2 R}$ & 12.1 & 1.14 & 34.0 & 7.18 \\
$U_{3}^{\mu}$ & 17.9 & 2.32 & 87.0 & 18.3 \\
$U_{1 L}^{\mu}$ & 10.1 & 0.505 & 16.2 & 1.91 \\
$U_{1 R}^{\mu}$ & 12.2 & 0.971 & 24.5 & 3.83 \\
$\widetilde{U}_{1}^{\mu}$ & 12.1 & 0.965 & 43.9 & 8.30 \\
$\sigma_{b g}$ & 8.14 & 0.0451 & 8.00 & 0.0285 \\
$\sigma_{W}$ & 68.3 & 1.05 & 68.5 & 0.928 \\
$\sigma_{Z}$ & 33.9 & 0.050 & 31.7 & 0.013 \\
\hline \hline
\end{tabular}

the region $|y|<3.5$. This cut reduces backgrounds like $t \bar{t}$ production which exhibit many more $e^{ \pm}$or jets in the central rapidity region.

(C4) The $e^{ \pm}$and jet should have $p_{T}>p_{T}^{\min }$ with $p_{T}^{\min }$ given in Table II.

(C5) We apply a cut on the missing $p_{T}$ requiring its value be lower than those in Table II.

(C6) Finally, we require that the cosine of the angles be-

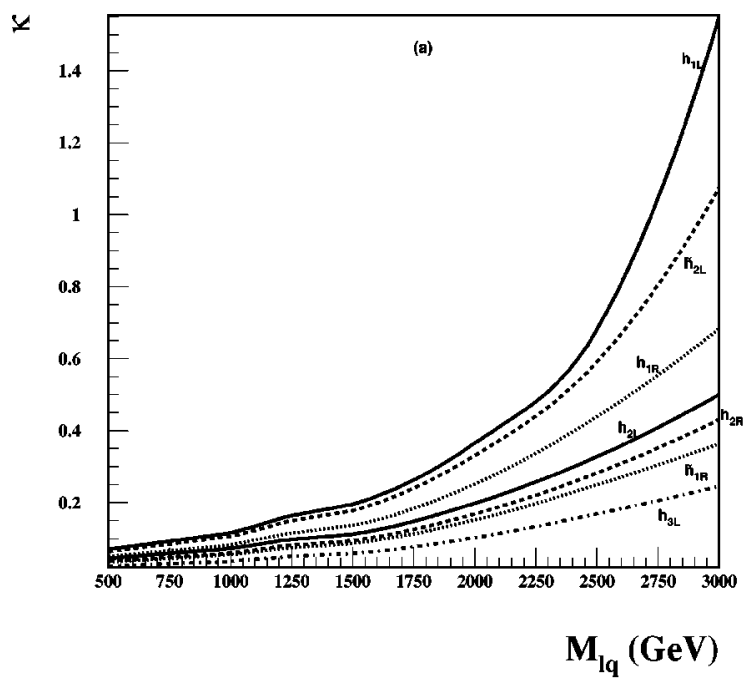

tween the direction of missing $p_{T}$ and the $p_{T}$ of $e^{ \pm}$and jet be larger than 0.94 .

In principle we should also require the $e^{ \pm}$to be isolated from hadronic activity in order to reduce the QCD backgrounds. Nevertheless, it was shown in Ref. [13] that this cut does not further suppress the background after we apply the cut (C4).

\section{RESULTS}

We present in Table III, as an illustration, the total cross section for producing pairs $e^{-}$-jet and $e^{+}$-jet, applying two different sets of cuts to the smeared final state momenta. We assumed in this table that $M_{\mathrm{lq}}=1 \mathrm{TeV}$ and $\kappa=0.3$. We denoted the irreducible $e q \rightarrow e q$ background by $\sigma_{b g}$, which was obtained setting $\kappa=0$. The cuts $(\mathrm{C} 3)-(\mathrm{C} 6)$ reduce the backgrounds by two or more orders of magnitude while the efficiency for the signal is of the order of 10-20\% depending on the leptoquark type. As we can see, the most important background is the $W$-jet production, which is larger then the $Z$-jet and irreducible backgrounds by a factor of roughly 20. Moreover, we verified using ISAJET [28] that the $t \bar{t}$ production background is effectively reduced by our cuts, especially (C3), it being negligible compared to the $W$-jet production. Therefore, the LHC reach in $\kappa$ and $M_{\mathrm{lq}}$ will be controlled by the $W$-jet background.

Leptoquarks of the type $F=2$ couple to pairs $e^{-} q$, and consequently are more copiously produced in $s$-channel processes leading to the final state $e^{-}$-jet than in reactions leading to $e^{+}$-jet since there are more quarks than antiquarks in the proton. For $F=0$ leptoquarks the situation is the opposite since they couple to $e^{-} \bar{q}$ pairs. As expected, the results shown in Table III agree with these arguments. Furthermore, using these features of $F=0$ and $F=2$ leptoquarks, we can differentiate between them simply by counting the number of leptoquark events with electrons and positrons in the final state.

In order to obtain the LHC attainable limits on lepto-

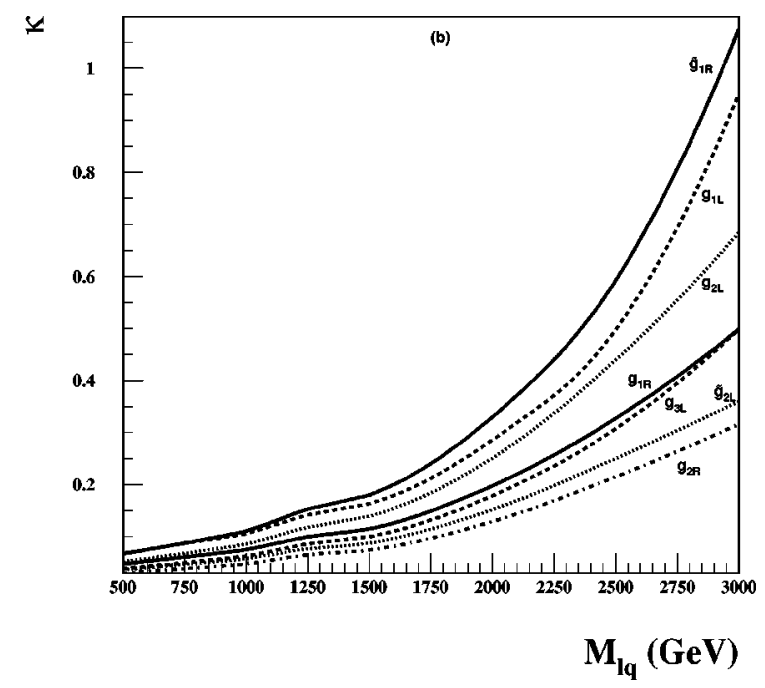

FIG. 5. $99.73 \%$ C.L. excluded regions in the $\kappa-M_{\mathrm{lq}}$ plane from negative searches of single production of leptoquarks with $F=0$ (a) and $F=2$ (b) for an integrated luminosity of $100 \mathrm{fb}^{-1}$. 


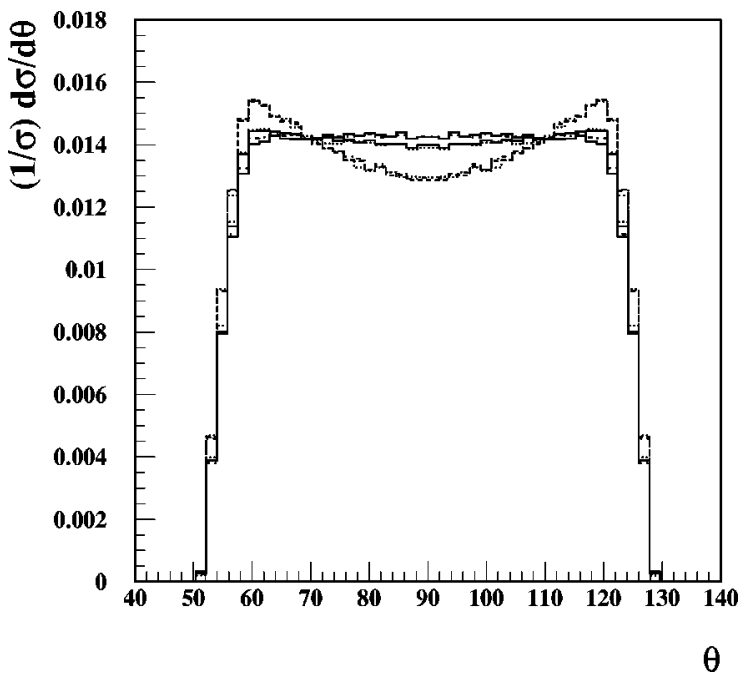

FIG. 6. Normalized polar angular distributions of the electron in the leptoquark rest frame, including the $W+$ jet background. We assumed that $\kappa=0.3$ and $M_{\mathrm{lq}}=1 \mathrm{TeV}$ and imposed the cuts (C1)(C6). The flatter lines correspond to the scalar $F=2$ leptoquarks while the peaked ones to the vector $F=2$ ones.

quarks we employed the final state $e^{-}$-jet $\left(e^{+}\right.$-jet $)$for $F$ $=2(F=0)$ leptoquarks since this topology possesses the largest signal cross section. Figures $5 \mathrm{a}$ and $5 \mathrm{~b}$ contain the regions in the plane $\kappa \times M_{\mathrm{lq}}$ that can be excluded at the 99.73\% C.L. ( $3 \sigma$ level) from negative single leptoquark searches at the LHC for an integrated luminosity of $100 \mathrm{fb}^{-1}$. As we can see, the LHC will be able to discover leptoquarks with masses of at least $2 \mathrm{TeV}$ for leptoquark Yukawa couplings of the electromagnetic strength $(\kappa$ $=0.3$ ). The $U_{3 \mu}$ leptoquarks will exhibit the tightest bounds while the $U_{1 \mu}$ leptoquarks will possess the loosest limits. Moreover, our results are comparable with those presented in

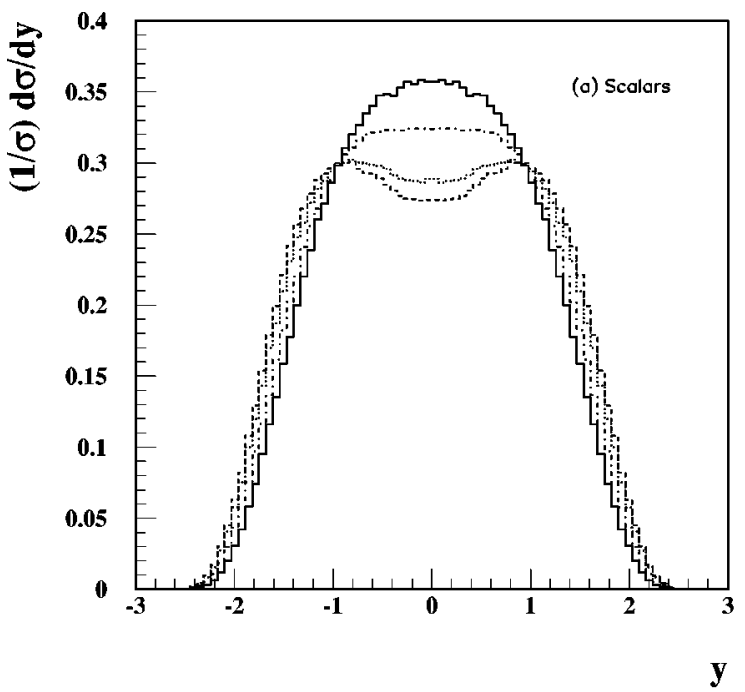

[13] for leptoquark searches using the processes (1)-(3).

We should also study the capability of the LHC to unravel the properties of leptoquarks in the event a signal is observed. As discussed above, the ratio of signal events in the channels $e^{+}$-jet and $e^{-}$-jet can discriminate between $F=0$ and $F=2$ leptoquarks. In order to learn more about the leptoquark giving rise to the signal, we should also study kinematical distributions. For instance, the $e^{ \pm}$polar angle distributions for scalars and vectors are distinct in the leptoquark rest frame. As an example, we show, in Fig. 6, the $e^{-}$normalized polar angle spectrum, including the backgrounds, for all $F=2$ leptoquarks. In this figure, we assumed $\kappa=0.3$ and $M_{\mathrm{lq}}=1 \mathrm{TeV}$ and applied the cuts (C1)-(C6). As expected, the distributions of scalar and vector leptoquarks are different, the scalar distribution being flatter. Nevertheless, the discovery of leptoquark spin will only be possible provided there will be enough events to render a statistical meaning to the angular distribution.

We can distinguish leptoquarks that couple to $u$ or $d$ quarks, analyzing the leptoquark pseudorapidity distribution in the laboratory frame because leptoquarks coupling to $u$ quarks are produced at larger rapidities than the ones coupling to $d$ 's. In Fig. 7, we show the normalized distributions after cuts for the pseudorapidity of scalar (a) and vector leptoquarks (b) with $F=2$, where the backgrounds were added to the signal. We can see three distinct curves in Fig. 7a: the largest distribution at central pseudorapidities is due to $\widetilde{S}_{1}$, which couples only to $d e^{-}$; the leptoquark triplet $S_{3}$ couples to $d e^{-}$and $u e^{-}$and gives rise to the curve in the middle; the curves peaked at higher rapidities originate from the production of $S_{1 R}$ and $S_{1 L}$ which couple exclusively to $e^{-} u$. Since $S_{1 R}$ possesses a larger branching ratio into $e^{-} u$ than $S_{1 L}$, its distribution is larger at high rapidities and less affected by the backgrounds. The situation is analogous for vector leptoquarks, as can be seen from Fig. $7 \mathrm{~b}$.

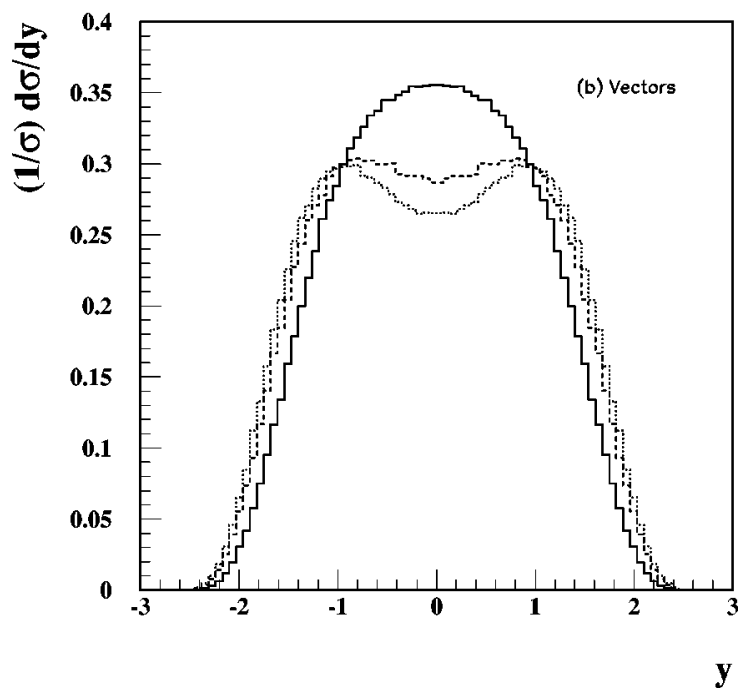

FIG. 7. Normalized pseudorapidity distributions of the leptoquarks, including the $W+$ jet background. We show the results for all types of leptoquarks with $F=2$, considering $\kappa=0.3$ and $M_{1 \mathrm{q}}=1 \mathrm{TeV}$. In (a) the solid line is for $\widetilde{S}_{1}$, the dashed line stands for $S_{1 R}$, the dotted line represents $S_{1 L}$, and the dash-dotted line is for $S_{3 B C}$. In (b) the solid line is for $V_{2 L}^{\mu}$, the dashed line represents $V_{2 R}^{\mu}$, and the dotted line stands for $\widetilde{V}_{2}^{\mu}$. 
TABLE IV. Attainable limits for the different leptoquark multiplets at $99.7 \%$ C.L. $(3 \sigma)$, assuming $\kappa=0.3$ and an integrated luminosity of $100 \mathrm{fb}^{-1}$.

\begin{tabular}{cc}
\hline \hline Leptoquark & $M_{1 \mathrm{qq}}(\mathrm{TeV})$ \\
\hline$S_{1 L}$ & 2.04 \\
$S_{1 R}$ & 2.40 \\
$\widetilde{S}_{1}$ & 1.92 \\
$S_{3 B C}$ & 2.47 \\
$V_{2 L}$ & 2.15 \\
$V_{2 R}$ & 2.92 \\
$\widetilde{V}_{2}$ & 2.73 \\
$R_{2 L}$ & 2.40 \\
$R_{2 R}$ & 2.56 \\
$\widetilde{R}_{2}$ & 1.92 \\
$U_{1 L}$ & 1.85 \\
$U_{1 R}$ & 2.14 \\
$\widetilde{U}_{1}$ & 2.73 \\
$U_{3 B C}$ & 3.21 \\
\hline \hline
\end{tabular}

\section{CONCLUSIONS}

The discovery of leptoquarks is without any doubt a striking signal for the existence of physics beyond the standard model. In this work we demonstrated that the search for leptoquarks in the process $p p \rightarrow e^{ \pm} q \rightarrow$ leptoquark $\rightarrow e^{ \pm} q$ at the LHC will be able to exclude leptoquarks with masses smaller than 2-3 TeV for Yukawa couplings of the order of the electromagnetic ones and an integrated luminosity of $100 \mathrm{fb}^{-1}$. Our results are summarized in Table IV. It is important to notice that our bounds are comparable to the ones coming from the reactions (1)-(3) [13]. Therefore, it will be possible to make a cross-check between the different channels and to improve the bounds combining them. Furthermore, the LHC will be able not only to confirm the present indirect limits on leptoquarks but also to expand them considerably.

If a leptoquark signal is observed at the LHC, we showed that we can determine whether the leptoquark is $F=0$ or $F$ $=2$ by counting the number of events in the $e^{-}$-jet and $e^{+}$-jet final states. Moreover, the spin of the leptoquark can also be established from the $e^{ \pm}$polar angle distribution in the leptoquark rest frame provided there are enough events for this distribution to be meaningful. We can even determine which leptoquark multiplet was produced by studying the rapidity spectrum of the leptoquarks in the laboratory frame. Finally, knowing the type of leptoquark we can estimate $\kappa$ using the size of the cross section, and consequently determine all leptoquark parameters. Once again, the pair and single production of leptoquarks via (1)-(3) can be used to confirm the leptoquark properties.

\section{ACKNOWLEDGMENTS}

One of us (O.J.P.E.) would like to thank the kind hospitality of the Institute for Elementary Particle Research, University of Wisconsin-Madison, where part of this work was done. P.G.M. would like to thank Xerxes Tata for useful discussion. This work was partially supported by Conselho Nacional de Desenvolvimento Científico e Tecnológico (CNPq), by Fundação de Amparo à Pesquisa do Estado de São Paulo (FAPESP), by the University of Wisconsin Research Committee with funds granted by the Wisconsin Alumni Research Foundation, and by the U.S. Department of Energy under Grants Nos. DE-FG02-95ER40896 and DEFG03-94ER40833.

\section{APPENDIX: SUBPROCESS CROSS SECTIONS}

Here we collect the nonpolarized differential cross sections in the center-of-mass frame for the processes $e^{ \pm} q(\bar{q})$ $\rightarrow e^{ \pm} q(\bar{q})$, including scalar and vector leptoquarks with $F$ $=0$ and $F=2$. The Feynman diagrams contributing to these reactions are displayed in Fig. 1. The differential cross section for the process $e^{-} q \rightarrow e^{-} q$ with the contribution of a $F=2$ scalar leptoquark is given by

$$
\begin{aligned}
& \left(\frac{d \hat{\sigma}}{d \cos \theta}\right)_{e^{-} q \rightarrow e^{-} q}^{S} \\
& =\frac{1}{32 \pi \hat{s}}\left[\left|\mathcal{M}_{\gamma}\right|^{2}+\left|\mathcal{M}_{Z^{0}}\right|^{2}+\left|\mathcal{M}_{S}\right|^{2}+2 \operatorname{Re}\left(\mathcal{M}_{\gamma Z^{0}}\right)\right. \\
& \left.\quad+2 \operatorname{Re}\left(\mathcal{M}_{\gamma S}\right)+2 \operatorname{Re}\left(\mathcal{M}_{Z^{0} S}\right)\right],
\end{aligned}
$$

with

$$
\begin{aligned}
& \left|\mathcal{M}_{\gamma}\right|^{2}=2 Q_{q}^{2} e^{4} \frac{\hat{s}^{2}+\hat{u}^{2}}{\hat{t}^{2}} \\
& \left|\mathcal{M}_{Z^{0}}\right|^{2}=2\left(G_{F} m_{Z^{0}}^{2}\right)^{2} \frac{1}{\left(\hat{t}-m_{Z^{0}}^{2}\right)^{2}} \\
& \times\left[\left(R_{e}^{2} R_{q}^{2}+L_{e}^{2} L_{q}^{2}\right) \hat{s}+\left(R_{e}^{2} L_{q}^{2}+L_{e}^{2} R_{q}^{2}\right) \hat{u}\right], \\
& \left|\mathcal{M}_{S}\right|^{2}=\frac{\kappa^{4}}{4} \frac{\hat{s}^{2}}{\left(\hat{s}-m_{S}^{2}\right)^{2}+m_{S}^{2} \Gamma_{S}^{2}}, \\
& 2 \operatorname{Re}\left(\mathcal{M}_{\gamma Z^{0}}\right)=-\frac{4 G_{F} m_{Z^{0}}^{2} Q_{q} e^{2}}{\sqrt{2}} \frac{1}{\hat{t}\left(\hat{t}-m_{Z^{0}}^{2}\right)} \\
& \times\left[\left(R_{e} R_{q}+L_{e} L_{q}\right) \hat{s}^{2}+\left(R_{e} L_{q}+L_{e} R_{q}\right) \hat{u}\right], \\
& 2 \operatorname{Re}\left(\mathcal{M}_{\gamma S}\right)=Q_{q} e^{2} \kappa^{2} \frac{\hat{s}^{2}\left(\hat{s}-m_{S}^{2}\right)}{\hat{t}\left[\left(\hat{s}-m_{S}^{2}\right)^{2}+m_{S}^{2} \Gamma_{S}^{2}\right]}, \\
& 2 \operatorname{Re}\left(\mathcal{M}_{Z^{0} S}\right)=-\frac{2 G_{F} m_{Z^{0}}^{2} \lambda_{L / R}^{2}}{\sqrt{2}} \frac{\hat{s}^{2}\left(\hat{s}-m_{S}^{2}\right)}{\left(\hat{t}-m_{Z^{0}}^{2}\right)\left[\left(\hat{s}-m_{S}^{2}\right)+m_{S}^{2} \Gamma_{S}^{2}\right]}
\end{aligned}
$$

We defined $\lambda_{L / R}$ 


$$
\begin{gathered}
\lambda_{L} \equiv \kappa_{L} \sqrt{L_{e} L_{q}}, \\
\lambda_{R} \equiv \kappa_{R} \sqrt{R_{e} R_{q}},
\end{gathered}
$$

with $R_{f}\left(L_{f}\right)$ being the right-handed (left-handed) coupling of the fermion $f$ to the $Z$, defined as

$$
\begin{aligned}
& L_{f}=2\left(T_{3}^{f}-Q_{f} \sin ^{2} \theta_{W}\right), \\
& R_{f}=-2 Q_{f} \sin ^{2} \theta_{W},
\end{aligned}
$$

where $Q_{f}$ is the electromagnetic charge of the fermion, $T_{3}^{f}$ is the third component of the isospin, and $\theta_{W}$ is the weak angle.

For $F=2$ vector leptoquarks, we have

$$
\begin{aligned}
& \left(\frac{d \hat{\sigma}}{d \cos \theta}\right)_{e^{-} q \rightarrow e^{-} q}^{V} \\
& =\frac{1}{32 \pi \hat{s}}\left[\left|\mathcal{M}_{\gamma}\right|^{2}+\left|\mathcal{M}_{Z^{0}}\right|^{2}+\left|\mathcal{M}_{V}\right|^{2}+2 \operatorname{Re}\left(\mathcal{M}_{\gamma Z^{0}}\right)\right. \\
& \left.\quad+2 \operatorname{Re}\left(\mathcal{M}_{\gamma V}\right)+2 \operatorname{Re}\left(\mathcal{M}_{Z^{0} V}\right)\right],
\end{aligned}
$$

with

$$
\begin{aligned}
\left|\mathcal{M}_{V}\right|^{2} & =\kappa^{4} \frac{\hat{u}^{2}}{\left(\hat{s}-m_{V}^{2}\right)^{2}+m_{V}^{2} \Gamma_{V}^{2}}, \\
\mathcal{M}_{\gamma V}= & 2 Q_{q} \kappa^{2} e^{2} \frac{\hat{u}^{2}\left(\hat{s}-m_{V}^{2}\right)}{\hat{t}\left[\left(\hat{s}-m_{V}^{2}\right)^{2}+m_{V}^{2} \Gamma_{V}^{2}\right]}, \\
2 \operatorname{Re}\left(\mathcal{M}_{Z^{0} V}\right)= & -\frac{4 \lambda_{L / R}^{\prime 2} G_{F} m_{Z^{0}}^{2}}{\sqrt{2}} \\
& \times \frac{\hat{u}^{2}\left(\hat{s}-m_{V}^{2}\right)}{\left(\hat{t}-m_{Z^{0}}^{2}\right)\left[\left(\hat{s}-m_{V}^{2}\right)^{2}+m_{V}^{2} \Gamma_{V}^{2}\right]},
\end{aligned}
$$

where

$$
\begin{gathered}
\lambda_{L}^{\prime} \equiv \kappa_{L} \sqrt{L_{e} R_{q}}, \\
\lambda_{R}^{\prime} \equiv \kappa_{R} \sqrt{R_{e} L_{q}} .
\end{gathered}
$$

The differential cross section of the process $e^{-} \bar{q} \rightarrow e^{-} \bar{q}$, taking into account the contribution of a $F=2$ scalar leptoquark, is

$$
\begin{aligned}
& \left(\frac{d \hat{\sigma}}{d \cos \theta}\right)_{e^{-} \bar{q} \rightarrow e^{-} \bar{q}}^{S} \\
& =\frac{1}{32 \pi \hat{s}}\left[\left|\mathcal{M}_{\gamma}\right|^{2}+\left|\mathcal{M}_{Z^{0}}^{\prime}\right|^{2}+\left|\mathcal{M}_{S}^{\prime}\right|^{2}\right. \\
& \left.\quad+2 \operatorname{Re}\left(\mathcal{M}_{\gamma Z^{0}}^{\prime}\right)+2 \operatorname{Re}\left(\mathcal{M}_{\gamma S}^{\prime}\right)+2 \operatorname{Re}\left(\mathcal{M}_{Z^{0} S}^{\prime}\right)\right],
\end{aligned}
$$

with $\mathcal{M}^{\prime}$ given by Eqs. (A3) and (A7), just switching $\hat{s} \leftrightarrow \hat{u}$ and $\left|\mathcal{M}_{\gamma}\right|^{2}$ given by Eq. (A2). The cross section for this process including vector leptoquarks is

$$
\begin{aligned}
& \left(\frac{d \hat{\sigma}}{d \cos \theta}\right)_{e^{-} \bar{q} \rightarrow e^{-} \bar{q}}^{V} \\
& =\frac{1}{32 \pi \hat{s}}\left[\left|\mathcal{M}_{\gamma}\right|^{2}+\left|\mathcal{M}_{Z^{0}}^{\prime}\right|^{2}+\left|\mathcal{M}_{V}^{\prime \prime}\right|^{2}+2 \operatorname{Re}\left(\mathcal{M}_{\gamma Z^{0}}^{\prime}\right)\right. \\
& \left.\quad+2 \operatorname{Re}\left(\mathcal{M}_{\gamma V}^{\prime \prime}\right)+2 \operatorname{Re}\left(\mathcal{M}_{Z^{0} V}^{\prime \prime}\right)\right],
\end{aligned}
$$

where $\mathcal{M}_{\gamma(Z) V}^{\prime \prime}$ are given by (A13) and (A15) with the change $\hat{s} \leftrightarrow \hat{u}$ and the other terms remain unchanged.

Now, we show the nonpolarized differential cross sections for $F=0$ scalar and vector leptoquarks. The cross section for the process $e^{+} q \rightarrow e^{+} q$ including a scalar leptoquark is

$$
\begin{aligned}
& \left(\frac{d \hat{\sigma}}{d \cos \theta}\right)_{e^{+} q \rightarrow e^{+} q}^{R} \\
& =\frac{1}{32 \pi \hat{s}}\left[\left|\mathcal{M}_{\gamma}\right|^{2}+\left|\mathcal{M}_{Z^{0}}\right|^{2}+\left|\mathcal{M}_{R}\right|^{2}+2 \operatorname{Re}\left(\mathcal{M}_{\gamma Z^{0}}\right)\right. \\
& \left.\quad+2 \operatorname{Re}\left(\mathcal{M}_{\gamma R}\right)+2 \operatorname{Re}\left(\mathcal{M}_{Z^{0} R}\right)\right],
\end{aligned}
$$

with $\left|\mathcal{M}_{\gamma}\right|^{2},\left|\mathcal{M}_{Z^{0}}\right|^{2}$, and $2 \operatorname{Re}\left(\mathcal{M}_{\gamma Z^{0}}\right)$ given by Eqs. (A2), (A3), and (A5), respectively, with the exchange $\hat{s} \leftrightarrow \hat{u}$. The remaining contributions are

$$
\begin{aligned}
\left|\mathcal{M}_{R}\right|^{2} & =\frac{\kappa^{4}}{4} \frac{\hat{s}^{2}}{\left(\hat{s}-m_{R}^{2}\right)^{2}+m_{R}^{2} \Gamma_{R}^{2}}, \\
2 \operatorname{Re}\left(\mathcal{M}_{\gamma R}\right) & =-Q_{q} e^{2} \kappa^{2} \frac{\hat{s}^{2}\left(\hat{s}-m_{R}^{2}\right)}{\hat{t}\left[\left(\hat{s}-m_{R}^{2}\right)^{2}+m_{R}^{2} \Gamma_{R}^{2}\right]},
\end{aligned}
$$

$$
\begin{aligned}
2 \operatorname{Re}\left(\mathcal{M}_{Z^{0} R}\right)= & \frac{2 G_{F} m_{Z^{0}}^{2} \eta_{L / R}^{2}}{\sqrt{2}} \\
& \times \frac{\hat{s}^{2}\left(\hat{s}-m_{R}^{2}\right)}{\left(\hat{t}-m_{Z^{0}}^{2}\right)\left[\left(\hat{s}-m_{R}^{2}\right)^{2}+m_{R}^{2} \Gamma_{R}^{2}\right]},
\end{aligned}
$$

with

$$
\begin{gathered}
\eta_{L}=\kappa_{L} \sqrt{L_{e} R_{q}}, \\
\eta_{R}=\kappa_{R} \sqrt{R_{e} L_{q}} .
\end{gathered}
$$

The cross section of the process $e^{+} q \rightarrow e^{+} q$ including $F$ $=0$ vector leptoquarks is 


$$
\begin{aligned}
\left(\frac{d \hat{\sigma}}{d \cos \theta}\right)_{e^{+} q \rightarrow e^{+} q}^{U} & \\
= & \frac{1}{32 \pi \hat{s}}\left[\left|\mathcal{M}_{\gamma}\right|^{2}+\left|\mathcal{M}_{Z^{0}}\right|^{2}+\left|\mathcal{M}_{U}\right|^{2}+2 \operatorname{Re}\left(\mathcal{M}_{\gamma Z^{0}}\right)\right. \\
& \left.+2 \operatorname{Re}\left(\mathcal{M}_{\gamma U}\right)+2 \operatorname{Re}\left(\mathcal{M}_{Z^{0} U}\right)\right],
\end{aligned}
$$

where

$$
\begin{aligned}
\left|\mathcal{M}_{U}\right|^{2}= & \kappa^{4} \frac{\hat{u}^{2}}{\left(\hat{s}-m_{U}^{2}\right)^{2}+m_{U}^{2} \Gamma_{U}^{2}}, \quad(\mathrm{~A} 27) \\
2 \operatorname{Re}\left(\mathcal{M}_{\gamma U}\right)= & -\frac{2 Q_{q} e^{2} \kappa^{2}}{3} \frac{\hat{u}^{2}\left(\hat{s}-m_{U}^{2}\right)}{\hat{t}\left[\left(\hat{s}-m_{U}^{2}\right)^{2}+m_{U}^{2} \Gamma_{U}^{2}\right]}, \\
2 \operatorname{Re}\left(\mathcal{M}_{Z^{0} U}\right)= & \frac{4 G_{F} m_{Z^{0}}^{2} \eta_{L / R}^{\prime 2}}{\sqrt{2}} \\
& \times \frac{\hat{u}^{2}\left(\hat{s}-m_{U}^{2}\right)}{\left(\hat{t}-m_{Z^{0}}^{2}\right)\left[\left(\hat{s}-m_{U}^{2}\right)^{2}+m_{U}^{2} \Gamma_{U}^{2}\right]} .
\end{aligned}
$$

We introduced the definitions

$$
\begin{gathered}
\eta_{L}^{\prime}=\kappa_{L} \sqrt{L_{e} L_{q}}, \\
\eta_{R}^{\prime}=\kappa_{R} \sqrt{R_{e} R_{q}} .
\end{gathered}
$$

Finally, the cross section of the process $e^{+} \bar{q} \rightarrow e^{+} \bar{q}$, taking into account $F=0$ scalar leptoquarks, is

$$
\begin{aligned}
\left(\frac{d \hat{\sigma}}{d \cos \theta}\right)_{e^{+} \bar{q} \rightarrow e^{+} \bar{q}}^{R} & \\
= & \frac{1}{32 \pi \hat{s}}\left[\left|\mathcal{M}_{\gamma}\right|^{2}+\left|\mathcal{M}_{Z^{0}}^{\prime}\right|^{2}+\left|\mathcal{M}_{R}^{\prime}\right|^{2}+2 \operatorname{Re}\left(\mathcal{M}_{\gamma Z^{0}}^{\prime}\right)\right. \\
& \left.+2 \operatorname{Re}\left(\mathcal{M}_{\gamma R}^{\prime}\right)+2 \operatorname{Re}\left(\mathcal{M}_{Z^{0} R}^{\prime}\right)\right],
\end{aligned}
$$

with $\left|\mathcal{M}_{\gamma}\right|^{2},\left|\mathcal{M}_{Z^{0}}^{\prime}\right|^{2}$, and $2 \operatorname{Re}\left(\mathcal{M}_{\gamma Z^{0}}^{\prime}\right)$ the same as in Eqs. (A2), (A3), and (A5), respectively, and $\left|\mathcal{M}_{R}^{\prime}\right|^{2}, 2 \operatorname{Re}\left(\mathcal{M}_{\gamma R}^{\prime}\right)$, and $2 \operatorname{Re}\left(\mathcal{M}_{Z^{0} R}^{\prime}\right)$ given by Eqs. (A21), Eq. (A22) by Eq. (A23), respectively, with the change $\hat{s} \leftrightarrow \hat{u}$.

For the vector leptoquarks, the cross section of this last process is

$$
\begin{aligned}
& \left(\frac{d \hat{\sigma}}{d \cos \theta}\right)_{e^{+} \bar{q} \rightarrow e^{+} \bar{q}}^{U} \\
& =\frac{1}{32 \pi \hat{s}}\left[\left|\mathcal{M}_{\gamma}\right|^{2}+\left|\mathcal{M}_{Z^{0}}^{\prime}\right|^{2}+\left|\mathcal{M}_{U}^{\prime \prime}\right|^{2}+2 \operatorname{Re}\left(\mathcal{M}_{\gamma Z^{0}}^{\prime}\right)\right. \\
& \left.\quad+2 \operatorname{Re}\left(\mathcal{M}_{\gamma U}^{\prime \prime}\right)+2 \operatorname{Re}\left(\mathcal{M}_{Z^{0} U}^{\prime \prime}\right)\right],
\end{aligned}
$$

with $\mathcal{M}^{\prime \prime}$ given by Eqs. (A27)-(A29), switching $\hat{s} \leftrightarrow \hat{u}$, and the other terms are the same as presented in Eq. (A32).
[1] S. Dimopoulos, Nucl. Phys. B168, 69 (1981); E. Farhi and L. Susskind, Phys. Rev. D 20, 3404 (1979); J. Ellis et al., Nucl. Phys. B182, 529 (1981).

[2] For a review see W. Buchmüller, Acta Phys. Austriaca, Suppl. 27, 517 (1985).

[3] L. Abbott and E. Farhi, Phys. Lett. 101B, 69 (1981); Nucl. Phys. B189, 547 (1981).

[4] See, for instance, P. Langacker, Phys. Rep. 72, 185 (1981).

[5] J. L. Hewett and T. G. Rizzo, Phys. Rep. 183, 193 (1989).

[6] O. J. P. Éboli and A. V. Olinto, Phys. Rev. D 38, 3461 (1988); J. L. Hewett and S. Pakvasa, ibid. 37, 3165 (1988); J. Blümlein, E. Boos, and A. Kryukov, Z. Phys. C 76, 137 (1997); M. Krämer et al., Phys. Rev. Lett. 79, 341 (1997); J. L. Hewett and T. Rizzo, Phys. Rev. D 56, 5709 (1997); T. Rizzo, Report No. SLAC-PUB-7284, hep-ph/9609267.

[7] W. Buchmüller, R. Rückl, and D. Wyler, Phys. Lett. B 191, 442 (1987).

[8] J. Wudka, Phys. Lett. 167B, 337 (1986); M. A. Doncheski and J. L. Hewett, Z. Phys. C 56, 209 (1992); J. Blümlein, E. Boos, and A. Pukhov, Mod. Phys. Lett. A 9, 3007 (1994); C. Friberg, E. Norrbin, and T. Sjöstrand, Phys. Lett. B 403, 329 (1997); Z. Kunszt and W. J. Stirling, Z. Phys. C 75, 453
(1997); J. Kalinowski et al., ibid. 74, 595 (1997); J. Blümlein, E. Boos, and A. Krykov, in "Proceedings of the 1996 HERA Physics Workshop,' hep-ph/9608483; T. Plehn et al., Z. Phys. C 74, 611 (1997); J. Blümlein, ibid. 74, 605 (1997).

[9] J. L. Hewett and T. G. Rizzo, Phys. Rev. D 36, 3367 (1987); J. L. Hewett and S. Pakvasa, Phys. Lett. B 227, 178 (1987); J. E. Cieza Montalvo and O. J. P. Éboli, Phys. Rev. D 47, 837 (1993); J. Blümlein and E. Boos, Nucl. Phys. B (Proc. Suppl.) 37, 181 (1994); C. G. Papadopoulos, Report No. DEMO-HEP97/04, hep-ph/9703372; J. Blümlein, E. Boos, and A. Krykov, Phys. Lett. B 392, 150 (1997); M. S. Berger, Report No. IUHET-343, hep-ph/9609517; M. A. Doncheski and S. Godfrey, Phys. Lett. B 393, 355 (1997); J. Blümein and R. Rückl, ibid. 304, 337 (1993).

[10] F. Cuypers, Int. J. Mod. Phys. A 11, 1627 (1996); F. Cuypers, P. H. Frampton, and R. Rueckl, Phys. Lett. B 390, 221 (1997).

[11] O. J. P. Éboli, E. M. Gregores, M. B. Magro, P. G. Mercadante, and S. F. Novaes, Phys. Lett. B 311, 147 (1993); H. Nadeau and D. London, Phys. Rev. D 47, 3742 (1993); M. A. Doncheski and S. Godfrey, ibid. 51, 1040 (1995); T. M. Aliev, E. Iltan, and N. K. Pak, ibid. 54, 4263 (1996); F. Cuypers, Nucl. Phys. B474, 57 (1996). 
[12] G. Bélanger, D. London, and H. Nadeau, Phys. Rev. D 49, 3140 (1994).

[13] A detail analyses of the signal and backgrounds for the processes (1)-(3) can be found in O. J. P. Éboli, R. Z. Funchal, and T. L. Lungov, Phys. Rev. D 57, 1715 (1998); E. Tsesmelis, ATLAS internal note PHYS-NO-029; A. d'Avella, ATLAS internal note PHYS-NO-026; B. Dion et al., Eur. Phys. J C2, 497 (1998).

[14] J. Ohnemus, S. Rudaz, T. F. Walsh, and P. M. Zerwas, Phys. Lett. B 334, 203 (1994).

[15] ALEPH Collaboration, D. Decamp et al., Phys. Rep. 216, 253 (1992); L3 Collaboration, O. Adraini et al., ibid. 236, 1 (1993); OPAL Collaboration, G. Alexander et al., Phys. Lett. B 263, 123 (1991).

[16] DELPHI Collaboration, P. Abreu et al., Phys. Lett. B 316, 620 (1993).

[17] S. Soldner-Rembold for the OPAL Collaboration, presented at the International Conference on Structure and Interactions of the Photon, Netherlands, 1997, hep-ex/9706003; DELPHI Collaboration, presented at the International Europhysics Conference Jerusalem, 1997, Report No. DELPHI 97-112 CONF 94.

[18] CDF Collaboration, F. Abe et al., Phys. Rev. Lett. 79, 4327 (1997); DØ Collaboration, B. Abbott et al., ibid., 79, 4321 (1997).

[19] ZEUS Collaboration, M. Derrick et al., Phys. Lett. B 306, 173 (1993); H1 Collaboration, I. Abt et al., Nucl. Phys. B396, 3
(1993); H1 Collaboratioan, S. Aid et al., Phys. Lett. B 369, 173 (1996).

[20] O. Shanker, Nucl. Phys. B204, 375 (1982).

[21] W. Buchmüller and D. Wyler, Phys. Lett. B 177, 377 (1986); J. C. Pati and A. Salam, Phys. Rev. D 10, 275 (1974).

[22] M. Leurer, Phys. Rev. Lett. 71, 1324 (1993); Phys. Rev. D 49, 333 (1994).

[23] P. Langacker, Phys. Lett. B 256, 277 (1991); P. Langacker, M. Luo, and A. K. Mann, Rev. Mod. Phys. 64, 87 (1992).

[24] G. Bhattacharyya, J. Ellis, and K. Sridhar, Phys. Lett. B 336, 100 (1994); 338, 522(E) (1994).

[25] O. J. P. Éboli, M. C. Gonzalez-Garcia, and J. K. Mizukoshi, Nucl. Phys. B443, 20 (1995); Phys. Lett. B 396, 238 (1997).

[26] S. Davidson, D. Bailey, and A. Campbell, Z. Phys. C 61, 613 (1994).

[27] P. Kessler, Nuovo Cimento 16, 809 (1960); V. Baier, V. Fadin, and V. A. Khoze, Nucl. Phys. B65, 381 (1973); M. Chen and P. M. Zerwas, Phys. Rev. D 12, 187 (1975); G. Altarelli and G. Parisi, Nucl. Phys. B126, 298 (1977).

[28] F. Paige and S. Protopopescu, in Supercollider Physics, edited by D. Soper (World Scientific, Singapore, 1986), p. 41; H. Baer, F. Paige, S. Protopopescu, and X. Tata, in Proceedings of the Workshop on Physics at Current Accelerators and Supercolliders, edited by J. Hewett, A. White, and D. Zeppenfeld (Argonne National Laboratory, Argonne, 1993). 